\title{
Investigation of ejection process in mechatronic functional modules of packaging machines
}

\section{Liudmyla Kryvoplias-Volodina, Oleksandr Gavva, Taras Hnativ, Kateryna Rivna}

\author{
National University of Food Technologies, Kyiv, Ukraine
}

Keywords:

Ejector

Mode

Packing

Machine

Simulation

\section{Article history:}

Received 04.02.2019

Received in revised form

11.07.2019

Accepted 30.09.2019

Corresponding author:

Liudmyla

Kryvoplias-Volodina

E-mail:

kryvopliasvolodina@

camozzi.ua

DOI: $10.24263 / 2304-$

974X-2019-8-3-16

\section{Abstract}

Introduction. The purpose of the research is to determine the influence of geometric, kinematic and technological parameters on the ejection processes in mechatronic functional modules of packaging machines.

Materials and methods. The modes of operation of pneumatic nozzle ejection systems with variable working environment were studied: compressed air, alcohol-containing solutions, gas-modified environment. The tasks set were solved on the basis of analysis and generalization of the literature material, conducting experiments to study the effectiveness of L-shaped ejectors, as well as bench studies of the process of creating and spraying the mixture on the product of processing.

Results and discussion. Problem solving for the proposed design of pneumatic nozzle ejection systems made possible to find optimal working technological and control modes for the processing of packing materials: dosed spraying of alcoholcontaining substances on the surface of treatment with back pressure within 3-5 bar, jet velocity at the inlet of the ejector is adjusted by pressure regulator, working area irrigation with a diameter of $100-150 \mathrm{~mm}$, controlling the effect of turning the ejector on and off by a variable feedback signal on the inlet pressure both in the ejector and in the pressure line with a liquid jet of $0-10 \mathrm{~V}$. Product processing above 6 bar causes the destruction of bakery products. For bakery products, the optimum pressure at the inlet of the ejector is 4 bars. Changing the geometry of the confuser and the diffuser makes it possible to reduce the flow of compressed air by $20 \%$, which reduces the energy load for packaging machines.

Conclusions. Therefore, the optimal working modes of pneumatic nozzle systems for the treatment of flexible packaging materials and the surface of non-destructive bakery products are proposed. 


\section{- Processes and Equipment-}

\section{Introduction}

In food packaging lines, when performing auxiliary technological operations, pneumatic nozzles devices with ejector systems are widely used $[1,12,18]$. On the basis of the analysis of the layout schemes of packaging machines, it was established that in a number of functional mechatronic mods (FMM) [1-3] are used with pneumatic systems. Examples of applications are surface coatings of packaging materials and consumer packaging, the introduction of a gas-modified medium (GMS) inside the package $[4,7,18]$, the separation of the flow of consumer packaging on the conveyor, the creation of a vacuum, cleaning of the carton valves before applying an adhesive solution, etc. In order to choose the rational design and operating modes of the ejector, taking into account the various physical and mechanical properties of food products, packaging materials - it is necessary to be able to simulate the kinematic and dynamic characteristics of the working environment during the ejection process $[16,17,18]$.

An effective method for modeling complex systems is simulation technology $[5,6,13]$. Which make it possible to take important technical solutions, accelerate the research and development processes, and shorten the time to create new packaging machines, while maintaining their competitive edge [8]. Solving the actual task of selecting and checking the rational parameters of ejectors in FMM packaging machines, - it is expedient to develop a generalized model for various working media (air, vacuum, GMS) $[9,15,17]$. Despite the rapid development of CFD-methods [10,14], the application of these methods does not always justify the expectations of the researcher. This is mainly due to the lack of a profile library of research environments and materials for CFD-engineers of scientific and production structures, as well as the lack of specialized literature $[11,16]$.

Literature analysis has shown that there was a lack of information on adjusting the energy costs of PM due to the use of feeders with gaseous media. It has also been found that most PM consist of FMM lifting the gas medium to the packing area without a feedback control signal, which makes it impossible to adjust the accuracy of the feed and change the spray area $[12,13,15]$. To adjust the functionality of packaging equipment with pneumatic nozzle ejector systems, it is important to expand the study of optimal technological characteristics of pneumatic nozzle ejectors.

In this regard, the actual purpose is to create methodological foundations for CFDcalculations of pneumatic nozzle in functional modules of packaging machines. In wellknown literary sources, particular attention is paid to the models of blown air and the cost characteristics of compressed air. In solving such problems, systematic analysis and synthesis of known literary sources was used [12], [13]. In view of this, the solution to the tasks of developing an ejector model and conducting a comparative analysis with theoretical studies of ejectors and the development of new structures is relevant, namely:

- development of CFD - model for reception of rational parameters of the ejector;

- synthesis of the ejector on the basis of the obtained results of imitative modeling;

- development of the structural control scheme of the ejector;

- substantiation of the proposed methodology for the analysis of pneumatic systems.

The analysis showed the need to study the structures and modes of operation of L-shaped ejectors to reduce their energy consumption in functional modules of packaging machines. There presented of the research is to study the effect of geometric, kinematic parameters on the technological characteristics of the proposed design of the pneumatic nozzle ejector in packaging machines. 


\section{- Processes and Equipment-}

\section{Materials and methods}

\section{Materials}

The object of the study is the hydrogasdynamic processes in the pneumatic nozzle ejector confuser-diffuser system. Particular attention is paid to changing the cost of the work environment and the recurrence of cycles to highlight the dose of the product $[11,14]$.

Despite the obvious advantages of semi-empirical methods over empirical methods, they have a number of disadvantages due to their limited scope and the need for a large amount of experimental data [16, 17]. As an alternative to empirical and semi-empirical methods, we have used approaches based on the solid-state mechanics, CFD methods (Computational Fluid Dynamics). That is, the system of Navier-Stokes or Reynolds equations is solved by numerical methods. $[3,7,8]$. The models developed can be used to analyze the operation of the packaging machine, to interpret the experimental results and to assist in the design and optimization of packaging lines. The research models were divided into two main categories:

1. Stable thermodynamic models that can be further divided into single-phase flow model and two-phase flow model;

2. Dynamic models, which are also divided according to phases [6,7].

Research materials include: compressed air, gas modified medium (GMC), packaging materials, alcohol solutions, pneumatic syringe systems, surface treatment operations, dosage systems, driver system. The design of the experimental ejector is designed taking into account the process of processing the surface of the packaging material during dosing-packing operations. The work of the ejector in the packaging machine for handling the packages is as follows. A high-pressure (working) gas with a full pressure $\mathrm{p}$ and a braking temperature $\mathrm{T}$ is brought through the coil 1 (Figure 1) into the mixing chamber 4. At the input channel of the

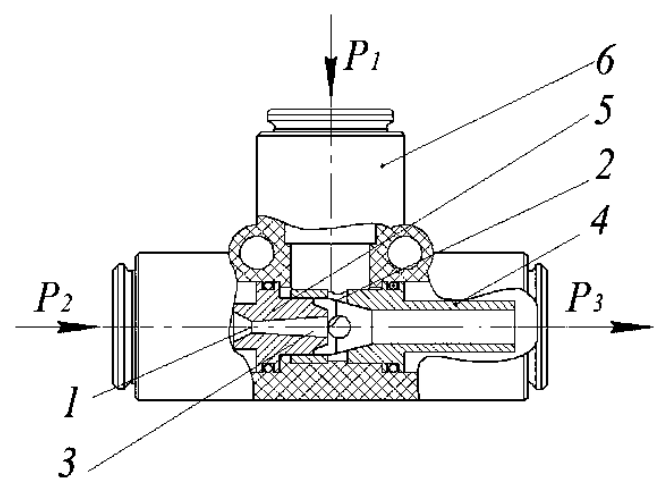

Figure 1. Scheme of a pilot ejector:

1 - channel of gas medium output by type of diffuser; 2 - sealing of the working zone of the vacuum; 3 - channel supply and narrowing of the gas medium by type of confuser; 4 - housing mixer chamber static pressure is set below the full pressure of the low pressure gas ejection.

Under the influence of the pressure difference, low pressure gas (or a working fluid close to the Newtonian) is directed to the mixing chamber. At the end of the chamber 4 after the completion of the mixing process, the gas has averaged parameters of the emulsion. The geometry of the L-shaped ejector is most widely used in packaging machines. When modeling in the environment of the PP Flow Vision, the main geometric parameter of the ejector is the ratio of the areas of the output nozzle sections for the working flow and the flow of ejection:

$$
\alpha=\frac{F_{1}}{F_{1}}=\frac{F_{1}}{F_{3}-F_{1}}
$$

where $F_{1}, F_{2}$ - respectively, the area of the outlet nozzle sections for supplying work flow and ejection flow; $F_{3}-$ the intersection area of the cylindrical mixing chamber (cross section at the outlet of the diffuser 4). 


\section{-Processes and Equipment-}

\section{Methods}

The paper used analytical and numerical methods of calculation with the setting of experimental development experiments. The result of a complex analytical calculation (selection of coefficients, refinement during the calculation of the ranges of the operating mode and geometric parameters, etc.), in the best case, integral parameters are obtained that are close to the experimental ones [5,6]. In the course of numerical simulation, it is possible to obtain not only adequate integral parameters, but also flow parameters (pressure, velocity, temperature, etc.) at any point in the computational domain. This is especially true when designing ejectors in which processes of mixing the working substances occur.

The methods for calculating ejectors contain various empirical coefficients, specially selected for a specific design and range of operation of the ejector. The range of application of the equations to describe the characteristics of the ejector is limited. Various methods of reducing the dimensions of the ejector design based on the use of a multi-nozzle apparatus for supplying an active stream are considered. Mixing chambers and nozzles, characterized by an L-like shape for packaging machines, are poorly understood $[8,9,11]$.

The calculation procedure is based on the use of numerical research results, namely, the obtained value of static pressure on the supply of working media to the ejector installation, followed by the assessment of changes in power and fuel consumption using analytical dependencies $[17,18]$. Numerical calculations of the structural and operational parameters of the ejector were carried out in a software package based on the finite element method. The calculations were performed in 2D and 3D statements as part of the solution of the stationary problem. In all calculations, the k- $\varepsilon$ RNG turbulence model with a standard near-wall function was used $[5,8,10]$. The criterion for the effectiveness of each ejector circuit is the pressure field at the outlet of the diffuser. The adequacy of computational studies was compared with experimental data obtained during experimental studies. Studies are aimed at optimizing the design of the ejector, namely, determining the optimal geometry of its individual nodes: an active vortex nozzle, a mixing chamber, a diffuser, and an internal air supply channel. The main goal was to reduce hydraulic losses along the path in the ejector elements for energy efficiency.

Research simulation metod. For a research simulation using a finite element method software package in Flow Vision [19] it is assumed that the ejector operates at a given static pressure at the outlet of the diffuser (for example, when it enters the atmosphere or inside a consumer packaging with constant pressure). The degree of expansion of the diffuser, was considered as a parameter that significantly affects the modes of the ejector. Confirmation of the adequacy of simulation models to the real process was carried out by experimental experiments on the developed stand. The parameters of the gas (working fluid) moving on the channel (nozzle system) can be changed by external influences, for example, due to heat supply, channel section changes, and the like. Often such physical actions are used to increase the flow rate of gas or increase in pressure. By the actions of external influence on pneumatic spray ejection systems are as follows:

1. Geometric - change in the cross-sectional area of the channel F.

2. Consumable - change of second consumption Ms.

3. Mechanical - supply or removal of mechanical energy $\mathrm{lm}$.

4. Teplovnye - supply or removal of heat qe.

5. Torting - the energy consumption of gas for friction lter.

6. Driver system - feedback control system.

The change (increase) of influences along the length of the channel is marked accordingly $-\mathrm{dF}$, dMc dlm, dqe, dlter. Moreover, all these changes, except for dlrr, can be both positive and negative. 


\section{Results and discussion}

\section{Integration of pneumatic nozzle ejectors in FMM packaging machine}

Analysis of the structure of packaging machines (PM) using a gas-containing medium in the package, showed the presence of complex FMM gas supply to the packaging area [5, 6]. Such modules are used in one layout with drives of linear and circular displacement [7, 9]. In order to expand the functionality of packaging equipment with pneumatic-emitting systems, it is necessary to provide for the search of rational technical solutions with optimal cost characteristics of FMM [8, 10, 11]. To improve the existing FMM by pneumatic-emitting systems for packaging food artificial and small-scale products, work was carried out with the involvement of simulation in PP Flow Vision [14, 15].

The basis of the arrangement of FMM with pneumo conducting ejection systems was selected PM for artificial products, processing of consumer packages and packing flexible materials based on ejectors with pneumatic or electropneumatic actuators for gas and Newtonian fluid media (technological environments). As a result of the topological analysis of the PM, a typical technological scheme of the FMM of the pneumatic plunger ejection systems is shown in Figure 2, which involves adjusting the magnitude of the effects on the ejector. For air blasting ejection systems, the work of which is directly related to the cost of compressed air and the working process environment, the system of precise direct control is related to the performance of a separate FMM and the entire packaging machine.

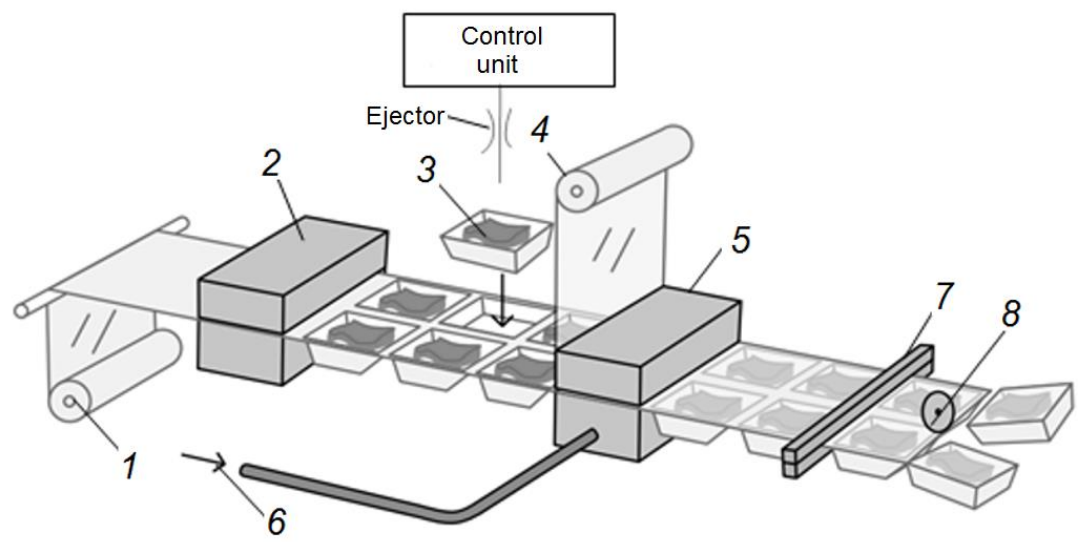

Figure 2. Technological scheme of PM for packing in a package of type "Sandwich":

1 - functional device for feeding the packaging material; 2 - formation of blister packaging; 3 - moving the product into a blister; 4 - feeding roll material for packing sealing; 5 - sealing blister; 6 - supply of gas mixture; 7 -the formation of transverse seams; 8 - cutting off the finished package

\section{Proposed control system}

During the study, the designs of the ejector of the packaging machine with control systems for dispensing the technological environment for food products were selected in Figure 3. Electropneumatic proportional control systems with the main technological parameters of the process were used. The greatest advantage is present in the arrangements with the electropneumatic automatic control system Figure 3. This involves connecting to the object of the regulating device (regulator) and input into the feedback system. The generalized block diagram is as follows: 


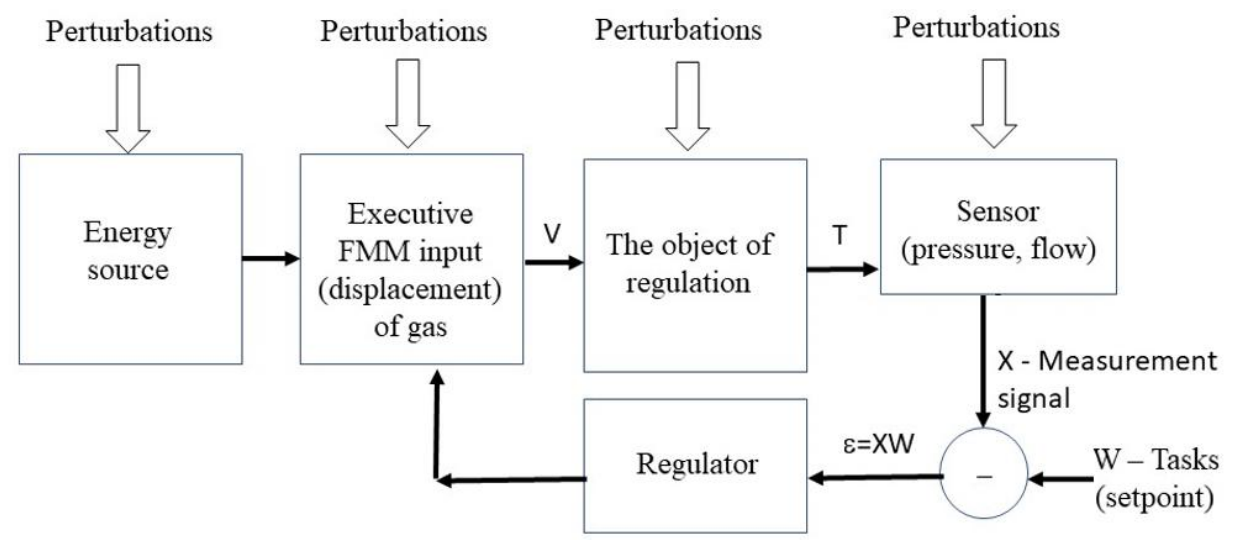

Figure 3. Block diagram of the automatic regulating system of the pneumatic nozzle FMM of the food packaging machine

The input of the regulator is the inconsistency signal $\varepsilon$ - the difference between the measured signal $\mathrm{X}$ and the set level W (setpoint). Despite the fact that they are part of the control system, the controller "does not know" the signals $\mathrm{V}$ and $\mathrm{T}$, directly related to the object. The task of the regulator is to control not only one object, but an entire system of three elements: an actuator, object, sensor. It complicates the management and reduces its quality. It turns out that the sudden expansion leads to hydraulic losses, that is, to loss of pressure (pressure), regardless of the losses caused by friction.

Development of a synthesis method for pneumatic nozzle ejection systems for FMM food packaging machines

Warehouse uncompressed Bernoulli equation for fluid that is when $\rho=$ const [2-7] :

$$
z_{1}+\frac{p}{\rho g}+\frac{w_{1}^{2}}{2 g}=z_{2}+\frac{p}{\rho g}+\frac{w_{2}^{2}}{2 g}+h_{\text {flow }} \quad z_{1}=z_{2}
$$

That is, the flow is horizontal, multiply the resulting expression on

$$
\rho g: p_{1}+\frac{\rho w_{1}^{2}}{2}=p_{2}+\frac{w_{2}^{2}}{g}+\rho g h_{\text {flow }} \quad \rho g h_{\text {flow }}=p_{\text {flow }}
$$

Get a loss of full pressure as a result of sudden expansion.

Apply the Euler equation

$$
p_{1}-p_{2}=\frac{\rho w_{2}^{2}}{2}-\frac{\rho w_{1}^{2}}{2}+p_{\text {flow }}
$$

$$
p_{1}-p_{2}=\rho w_{2}\left(w_{1}-w_{2}\right), \text { where } p_{1}-p_{2}
$$

loss of statistical pressure resulting from fluid movement from a high-pressure area to a site with less pressure. Since the plot $1-2$ is short, then the loss of liquid is not taken into account by the Carnot-Bordeaux theorem:

$$
\begin{gathered}
\rho w_{2}\left(w_{2}-w_{1}\right)=\frac{\rho w_{2}^{2}}{2}+\frac{\rho w_{1}^{2}}{2}+p_{\text {flow }}, \\
p_{\text {flow }}=\frac{\rho w_{1}^{2}}{2}-\rho w_{1} w_{2}+\frac{\rho w_{2}^{2}}{2}=\rho \frac{\left(w_{2}-w_{1}\right)^{2}}{2}, \\
p_{\text {flow }}=\frac{\rho}{2}\left(w_{1}-w_{2}\right)^{2} .
\end{gathered}
$$




\section{- Processes and Equipment-_}

With the help of this equation, you can make recommendations for eliminating the transition of sections of cylindrical cross sections with a smaller diameter to sections with large diameters on the coordinate system of the flow of the product. In a food dispenser close to rheology to Newtonian liquids, we have developed pneumorphic gas ejectors $[3,5,9]$. The resulting structure can be synthesized in functional mechatronic modules of packaging machines and used for moving gas or low pressure fluid through a high pressure gas jet.

\section{Investigation of the flow structure in the area of mixing jets}

The 3D ejector (Figure 4) consists of a nozzle 3 for high pressure gas (active or ejectable), 4 - nozzles for low pressure gas (passive or ejector), 1 - mixing chamber and 2 diffuser. The active gas stream, having a high velocity at the outlet of the nozzle 1 , and consequently, low pressure sucking from the nozzle 2 of the liquid (gas), and mixing with it in the chamber 3, transmits to it a portion of the energy that gives the mixture a certain speed. The nozzle 1 may be subsonic or supersonic (depends on the pressure drop and nozzle construction). Supersonic ejectors are more effective.
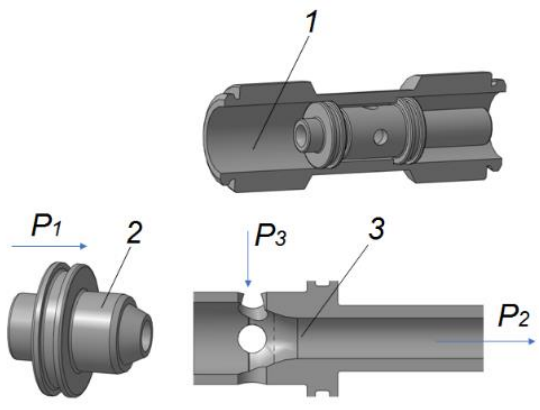

Figure 4. Developed ejector for surface treatment of food products: 1 - housing; 2 - diffuser; 3 - confuser

A feature is the change in the mass of a liquid product or gas moving at a speed $\mathrm{w} 1$ in the nozzle and w2 in the mixer chamber. The active jet injected into the ejector changes its speed from w1 to w2 speed in the mixer chamber. Changing the speed of the active jet MA $\left(\mathrm{w}_{2}-\mathrm{w}_{1}\right)$. Assume that the passive jet at the input to the ejector has a speed of $w=0$. In the mixer chamber its speed becomes w2. Changing the velocity of the passive jet $\mathrm{Mp}\left(\mathrm{w}_{2}-\mathrm{w}_{1}\right)$. Since there are no external forces acting on the gas, the total change in the amount of motion in accordance with the assumptions made is zero:

$$
\begin{aligned}
& \frac{M_{P}}{M_{A}}=\frac{w_{1}-w_{2}}{w_{1}}=n \\
& \frac{M_{P}}{M_{A}}=1-\frac{w_{2}}{w_{1}}=n
\end{aligned}
$$

As a result of the simulation, the tasks were set: to evaluate the flow characteristics of the proposed ejector model; determine the forces acting on the wall of the diffuser and the nozzle in the subsonic and sound modes of the ejectors in the dispenser; get results on the distribution of forces acting on the liquid (product) along the axis of motion in the projection on the horizontal axis. Accepted assumptions in the model: Gas flows, under some pressure. It creates the force of $\mathrm{RD}$, acting from the inside of the diffuser wall, perpendicular to its surface. Assume that this force acts on a diffuser at some point. In turn, on the wall of the diffuser, the reactive power of the WG is applied to the gas, applied to the control surface of the gas. In fact, as you know, the point of applying force and reactive force coincide. PG force can be divided into two. Vertical component of R Du, "disperses" the diffuser and is perceived by the material of the wall. A similar vertical force of an OC is R Du, acting on gas, is counterbalanced by the force acting on the gas from the side of the diffuser on the opposite side. The results are shown in table 1. 
Table 1

Results of the influence of changes in the geometric characteristics of the nozzle on the kinematic parameters of the system

\begin{tabular}{|c|c|c|c|c|c|}
\hline Mode & Cut 1 & $\begin{array}{c}\text { Cut } \\
\text { «narrowing» }\end{array}$ & Cut 2 & $\begin{array}{l}\text { Channel } \\
\text { function }\end{array}$ & Addiction \\
\hline 1 & 2 & 3 & 4 & 5 & 6 \\
\hline I & $\begin{array}{c}\mathrm{M}>1 \\
\mathrm{~W}<w_{c r} \\
\mathrm{P}>P_{c r}\end{array}$ & $\begin{array}{c}\mathrm{M}<1 \\
\mathrm{~W}<w_{c r} \\
\mathrm{P}>P_{c r}\end{array}$ & $\begin{array}{l}\mathrm{W}<w_{c r} \\
\mathrm{P}>P_{c r}\end{array}$ & $\begin{array}{l}\text { Venturi } \\
\text { tube }\end{array}$ & $\begin{array}{c}\text { In "narrowing" } \\
\text { speed does not reach } \\
\text { critical value }\end{array}$ \\
\hline II & $\begin{array}{c}\mathrm{M}<1 \\
\mathrm{~W}<w_{c r} \\
\mathrm{P}>P_{c r}\end{array}$ & $\begin{array}{c}\mathrm{M}=1 \\
\mathrm{w}=w_{c r} \\
\mathrm{P}>=P_{c r}\end{array}$ & $\begin{array}{c}\mathrm{W}>w_{c r} \\
\mathrm{P}<P_{c r}\end{array}$ & $\begin{array}{c}\text { Laval's } \\
\text { nozzle }\end{array}$ & $\begin{array}{l}\text { In "narrowing" speed } \\
\text { reaches critical and } \\
\text { growing rapidly }\end{array}$ \\
\hline III & $\begin{array}{c}\mathrm{M}>1 \\
\mathrm{~W}>w_{c r} \\
\mathrm{P}<P_{c r}\end{array}$ & $\begin{array}{c}\mathrm{M}=1 \\
\mathrm{w}=w_{c r} \\
\mathrm{P}=P_{c r}\end{array}$ & $\begin{array}{c}\mathrm{w}<w_{c r} \\
\mathrm{P}>P_{c r}\end{array}$ & $\begin{array}{l}\text { Supersonic } \\
\text { diffuser }\end{array}$ & $\begin{array}{c}\text { The rapid } \\
\text { increase } \\
\text { in pressure }\end{array}$ \\
\hline IV & $\begin{array}{c}\mathrm{M}>1 \\
\mathrm{~W}>w_{c r} \\
\mathrm{P}<P_{c r}\end{array}$ & $\begin{array}{l}\mathrm{M}<1 \\
\mathrm{~W}<w_{c r} \\
\mathrm{P}>P_{c r}\end{array}$ & $\begin{array}{c}\mathrm{W}>w_{c r} \\
\mathrm{P}<P_{c r}\end{array}$ & $\begin{array}{l}\text { Difficult to } \\
\text { implement }\end{array}$ & $\begin{array}{c}\text { A shock wave arises and } \\
\text { the gas flow turns into } \\
\text { subsonic }\end{array}$ \\
\hline
\end{tabular}

According to the modes of Table 1, it can be argued that one pneumatic nozzle channel can act as a Laval nozzle, a supersonic diffuser and a Venturi tube, depending on the parameters of the FMM working system at the inlet and outlet of the spray device. Therefore, to develop their own design of pneumatic nozzle ejecting device, the assumption is made: $\mathrm{F} 1=\mathrm{F} 2$; the movement of the gas inside the nozzle is energy-isolated, isoentropic and stationary, the system operates with purified air under normal conditions.

\section{Experimental design pneumonozzle system}

Thus, the greater the velocity of the active gas and the less passive, the greater the rate of ejection. As can be seen from the CFD model, Figure 5, this calculation is very simplistic.

The methodology of the study is shown in Figure 5, with a detailed description of the input parameters of modeling, step-by-step evaluation of mathematical models, comparative characteristics of the obtained results, approaches to the choice of rational parameters to build a physical model of pneumatic nozzle ejection system in Figure 6.

As a result of modeling, in contrast to the well-known research approaches $[5,8,11$, 14], we take into account the OEE - as a general criterion for evaluating the operation of a pneumatic nozzle ejector in FMM packaging machine. 


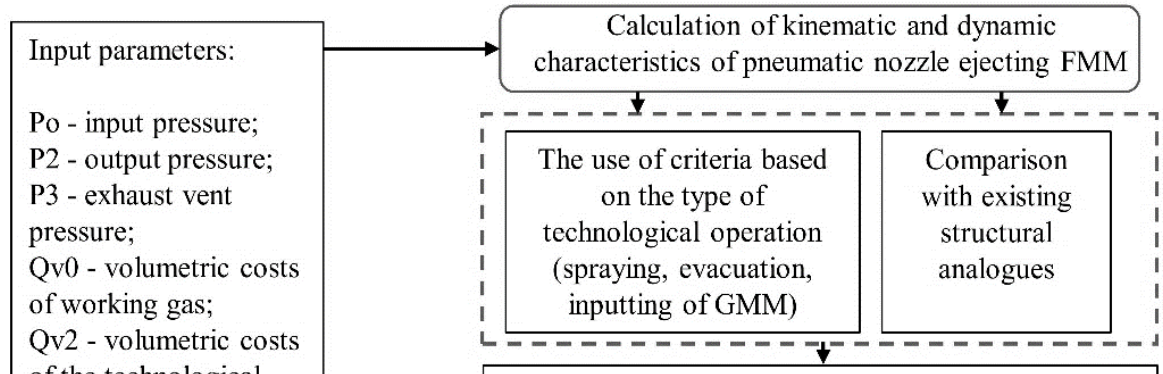

of the technological working environment; $\mathrm{Fi}$ - the area of the working sections; $\mathrm{k} ; \mathrm{R} ; \mu$ - the area of the working gas.

Determination of gas-dynamic characteristics of the working nozzle of the ejector

Determination of the law and method of control of the pneumatic nozzle system

Optimization of technological characteristics from the condition of generalized characteristics of influence on the velocity, pressure, density, temperature, flow

Calculation of dynamic characteristics of transients in the control system. Assessment of stability and quality of transients.

Numerical (simulation model) calculation of the load of the nozzle system on pressure, costs

Unsatis
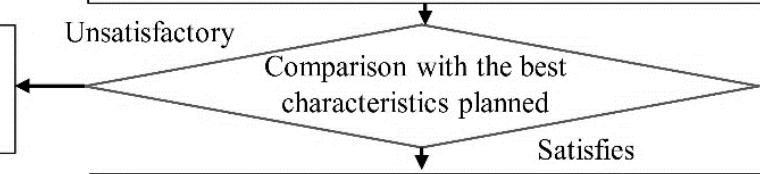

Refinement of the geometry of the internal section of the ejector, taking into account the $3 \mathrm{D}$ model

Calculation of working sections to clarify the simulation test model

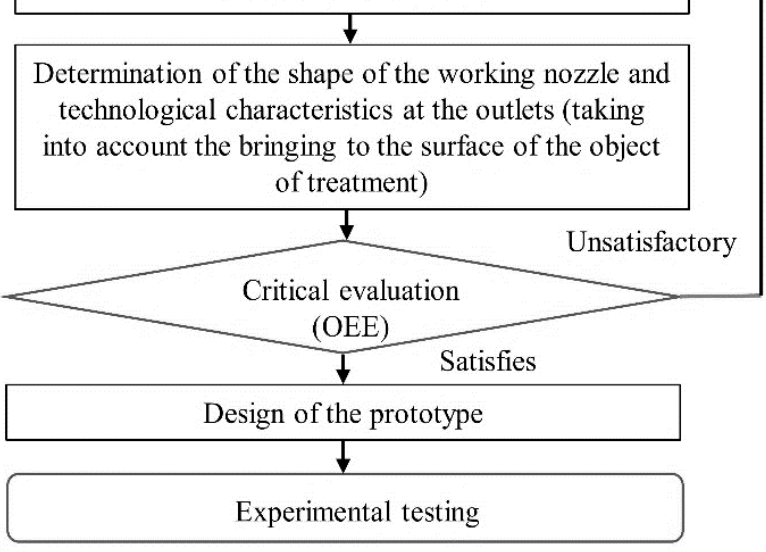

Figure 5. Structure of the algorithmic method of synthesis of a pneumatic plunger ejection functional mechatronic module for a packing machine 
To determine the force acting on the wall of the diffuser or nozzle with subsonic motion and the absence of hydraulic losses. Consider the forces acting on the gas (liquid) along the axis of motion in the projection onto the horizontal axis (Figure 6, a).

General scheme in the expected load on the wall pressure diffuser, (Figure 6,b):

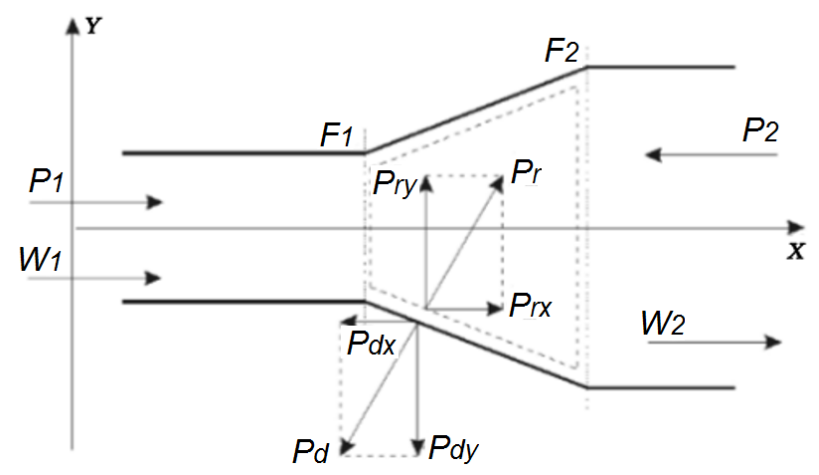

$a$

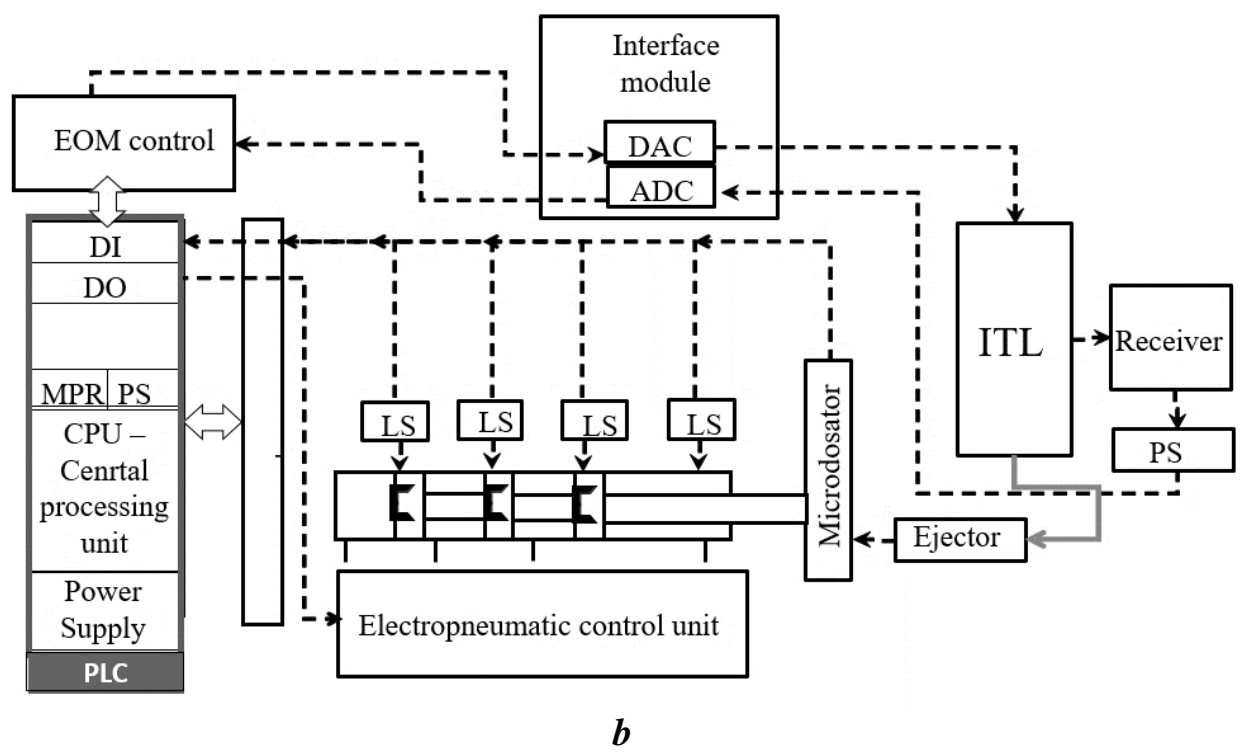

Figure 6. Analysis of the experimental system:

a-general scheme of the expected load on the wall of the pressure diffuser for the developed model ejector;

b - General scheme of the dispenser of the pneumo-ejecting system with supplying the spray jet to the FMM of the packaging machine:

DI - discrete inputs; DO - discrete outputs; MPR - mechatronic pressure regulator (analog inputs / analog outputs), proportional to the input signal; ITL - information transmission line; PS - pressure sensor; LS - location sensor; DAC - digital-to-analog converter; ADC - analog-to-digital converter. 


\section{-Processes and Equipment-}

The horizontal components of the RSU and Rd are not balanced. These forces are created by pressure of gas P1 from left to right in section 1 and pressure P2 from right to left in section 2. These pressures arise from the mass of gas surrounding the allocated volume. Pressure p2 prevents gas propagation in the direction of its movement from left to right

$$
P_{1}=p_{1} F_{1} ; P_{2}=p_{2} F_{2} \text {, }
$$

where: F1 and F2 are the cross-sectional areas of the input and output. As a result of the analysis of the developed ejector model, results have been obtained that prove that the dynamic models have a higher accuracy of prediction and provide more information in comparison with stable thermodynamic models.

For research, a special stand has been designed and manufactured on which it is possible to simulate different modes of operation of the piston microdoser within FMM processing of consumer packaging.

The block diagram of the experimental stand is shown in (Figure 6b).

When testing the stand for the operation of the piston micrometer, the tolerance limit for the nominal weight of the liquid product dose is $50 \mathrm{ml}$, not more than $1.5 \%$.

The transmission ratio for the pressure regulator is calculated by the formula:

$$
\mathrm{K}=\mathrm{P} / \mathrm{U}=9 \cdot 10^{4}(\mathrm{~Pa} / \mathrm{W}) \text {. }
$$

DAC with $\mathrm{U}=0 . .10 \mathrm{~V}$ output signal was used to control the MRI. The DAC channel transmission ratio is the ratio of the output voltage to the input code R'will be: $K^{\prime}=U^{\prime} / R^{\prime}=$ $1 / 409.6$ (V / disc unit). The minimum value of the pressure at the fixing corresponds to the signal of the reference $\Delta \mathrm{R}=1$ disc. Will be: $\Delta \mathrm{P}=\Delta \mathrm{R} \cdot \mathrm{K}^{\prime} \cdot \mathrm{K}=1 \cdot(10 / 4096) \cdot(900000 / 10)=220 \mathrm{~Pa}$. This value of $\Delta \mathrm{P}$ is $0.022 \%$ of the range of change in initial pressure, that is, sufficient from the point of view of the accuracy of pressure measurements during the experiments. The controls for the micro-dosing module were formed according to sinusoidal and stepped laws, using the software FluidLab-P, Version 1.0. SWCN-P-10 (Camozzi) was used to obtain information on the pressure values of the product receiver.

The study monitored the stability of the pressure in the receiver, which depends on the error of dosing at the exit of the microdosage. The general view and drawings of the design of the ejector microdosage are shown in Figure 6. The general view of the experimental stand with FMM supplying the microdose in the area of packaging and processing of consumer packaging.

The results of experimental studies have established the nature and duration of transient regulation of the pressure at the outlet of the MRI, and its subsequent impact on dose separation from the receiver. The amount of product formed and the velocity of the jet at the outlet of the nozzle portion of the dispenser depend on the regulation of the inlet pressure, the throughput of the working channel, and the operating pressure in the control part of the module. The characteristics of the control signal change are shown in (Figure 7).

The following results of the deviation of the repetition of the dose of the product were obtained, with the operation of the FMM microdoser according to the sinusoidal law of filing for spraying:

- product - Dewar`s12 (wiskey) - density $942 \mathrm{~kg} / \mathrm{m}^{3}$; diameter of the pipeline $0,004 \mathrm{~m}$, pressure $-1,8$ bar, pressure amplitude $1,2 \mathrm{bar}$; frequency varies within: $18-36 \mathrm{~Hz}$; deviation of the product dose within 2,2\% (Figure 7, a);

- product - Southern Comfort (liqueur) - density $994 \mathrm{~kg} / \mathrm{m}^{3}$; diameter of pipeline $0,004 \mathrm{~m}$, pressure $-1,8$ bar, pressure amplitude 1,2 bar; frequency varies within: $18-36 \mathrm{~Hz}$; deviation og the product dose within $1,5 \%$ (Figure $7, \mathrm{~b}$ );

- product - Monin (syrup) - density $1300 \mathrm{~kg} / \mathrm{m}^{3}$; diameter of pipeline $0,004 \mathrm{~m}$, pressure 1,8 bar, pressure amplidute 1,2 bar; frequency varies within: $18-36 \mathrm{~Hz}$; deviation of the product dose within $1,0 \%$ (Figure $7, \mathrm{c}$ ). 

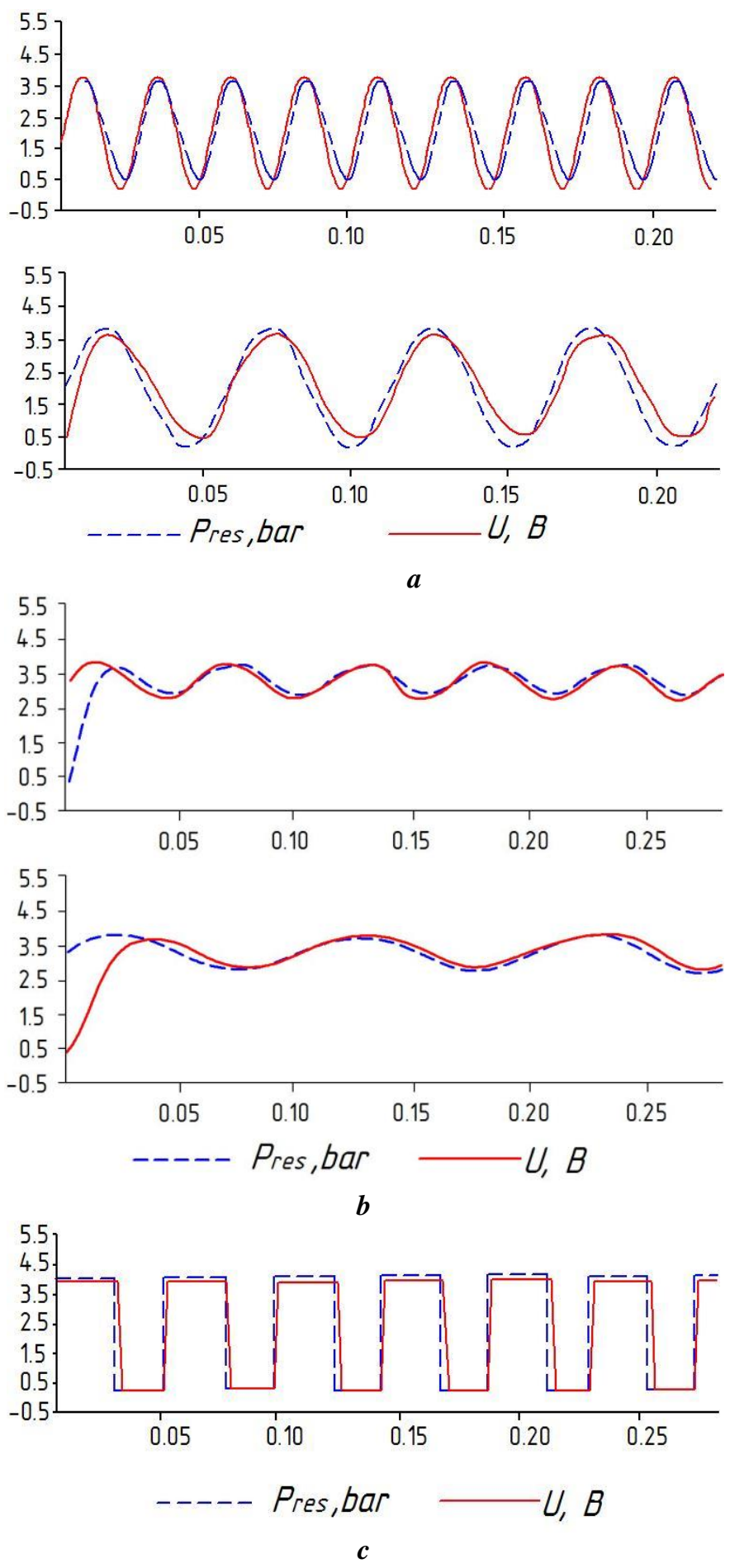

Figure 7. Generalized characteristic of the FMM microdosage

Ukrainian Food Journal. 2019. Volume 8. Issue 3- 631 


\section{-Processes and Equipment-}

\section{Conclusions}

1. When the correction parameters of the differential pressure feedback and its derivatives with the external mechatronic pneumatic controller in the microdetector are entered improved performance of the dosing module is obtained. The error in the formation of the dose of the product is significantly reduced and does not exceed the rules on the regulation of the technological process in packaging machines.

2. Based on the results of a series of simulation experiments, one can assume that the defining elements of the ejector's operating characteristics are:

- a nozzle that provides the required flow;

- mixing camera with the necessary parameters;

- the location of the nozzle and its form of communication with the surface of the ejector, providing the necessary pressure distribution and turning the jet.

3. The results of the simulation experiment confirmed the correspondence of the coupling model between pressure and velocity, presented in the course of the mathematical description. The obtained data showed a sharp change in the nature of the distribution of pressure and velocity inside ejection systems when changing the type of working environment in single-phase pneumatic systems. This is one of the criteria to take into account when choosing the type of control system of the FMM of the packaging machine.

4. Problem solving for the proposed design of pneumatic nozzle ejection systems made it possible to find optimal working technological and control modes for the processing of packing materials: dosed spraying of alcohol-containing substances on the surface of treatment with back pressure within 3-5 bar, jet velocity at the inlet of the ejector is adjusted by pressure regulator, working area irrigation with a diameter of $100-150 \mathrm{~mm}$, controlling the effect of turning the ejector on and off by a variable feedback signal on the inlet pressure both in the ejector and in the pressure line with a liquid jet of $0-10 \mathrm{~V}$.

5. Product processing above 6 bar causes the destruction of bakery products. For bakery products, the optimum pressure at the inlet of the ejector is 4 bars. Changing the geometry of the confuser and the diffuser makes it possible to reduce the compressed air flow rate by $20 \%$, which reduces the energy load for packaging machines. The digital controllers and flowmeter in the ejector system with $0-10 \mathrm{~V}$ feedback enable $0.1-0.5 \mathrm{~s}$ to change the performance of a separate functional mechatronic module of the packaging machine.

6. The 3D simulation model of a gas or liquid displacement ejector using a high pressure gas jet was synthesized. Deviation of the repeat dose of the product, when sprayed on biscuits: Dewar`s12 (whiskey) frequency varies within: $18-36 \mathrm{~Hz}$; product dose variation within $2.0 \%$; product - Southern Comfort (liqueur), product dose deviation within $1.3 \%$.

\section{References}

1. Smith J., Ramaswamy H., Simpson B. (1990), Developments in food packaging technology, Part II. Storage aspects, Trends Food Sci Tech, 1, pp. 111-118.

2. Vanderroost M., Ragaert P., Devlieghere F. (2017), Intelligent foodpackaging, The nextgeneration, Trends Food Sci Technol, 39, pp. 47-62.

3. Steven T. (2017), Signals and systems with Matlab applications, Orchard Publications. 


\section{- Processes and Equipment-}

4. Sun D-W, Emes IW. (1996), Performance characteristics of HCFC-123 ejector refrigeration cycles, Int. J. Energy Res, 20, pp. 871-885.

5. Sun D-W. (1997), Experimental investigation of the performance char-acteristics of a steam jet refrigeration system, Energy Sources, 19, pp. 349-367.

6. Takashi H. (2014), Inventor Japanese patent 2113849, Tempix The Tempix temperature indicator, Available at: http://tempix.com/.

7. Todorovic V., Neag M., Lazarevic M. (2014), On the Usage of RFID Tags for tracking and monitoring of shipped perishable goods, Procedia Engineering, 69, pp. 1345-1349.

8. Smith J., Hoshino J., Abe Y. (1995), Interactive packaging involving sachet technology, In. Rooney ML (ed) Active food packaging, Blackie Academic and Professional, London, pp. 143-173.

9. Kryvoplias-Volodina L., Gavva O., Volodin S., Hnativ T. (2018), Dynamics of mechatronic function modules drives of flow technological lines in food production, Ukrainian Journal of Food Science, 7(4), pp. 726-738.

10. Khalil A., Fatouh M., Elgendy E. (2011), Ejector design and theoretical study of R134a ejector refrigeration cycle, Int. J. Refrig., 34(7), pp.1684-1698.

11. Gavva O., Kryvoplias-Volodina L., Kohan E. (2014), Intensification of orientation for packing-case loads in machines that form packages, Journal of Food and Packaging Science Technique and Technologies, 3, pp. 111-116.

12. Zielinski A.P., Sanecki H., Karas M. (2017), Effectiveness of the Trefftz method in different engineering optimization procedures, CAMES, 8, pp. 479-493.

13. Wang, Quing-Guo, (2016), PID Control for Multuvariable Processes, ChangChieh Hang, Springer, P.262.

14. Yukimaru S., Shogo N., Sahao K., Shigemitsu K. (1987), Studies of the configuration and performance of annular type ejectors, Transactions ASME, $J$ Fluids Eng, 109, pp. 205-212.

15. Zagory D. (2015), Ethylene-removing packaging, In. Rooney ML (ed) Active food packaging, Blackie Academic and Professional, London, pp. 38-54.

16. Zhu Y., Cai W., Wen C., Li Y. (2007), Shock circle model for ejector performance evaluation, Energy Conversion and Management, 48, pp. 2533-2541.

17. Wessling C., Nielsen T., Leufven A., Jagersstad M. (1998), Mobility of $\alpha-$ tocopherol and BHT in LDPE in contact with fatty food stimulants, Food Addit Contam, 15, pp. 709-715.

18. Gavva O., Kryvoplias-Volodina L., Derenivska A. (2013), Ways to reduce dosing error of granular products in linear weight dosing machines, Journal of Food and Packaging Science Technique and Technologies, 2, pp. 232-236.

19. Acsenov A. (2017), FlowVision: Industrial computational fluid dynamics, Computer Research and Modeling, 9, pp. 5-20. 